\title{
Solvable and nilpotent right loops
}

\author{
Vipul Kakkar \\ School of Mathematics, \\ Harish-Chandra Research Institute, \\ India \\ email: vplkakkar@gmail.com
}

\author{
Vivek Kumar Jain \\ Department of Mathematics, \\ Central University of South Bihar, India \\ email: jaijinenedra@gmail.com
}

\begin{abstract}
In this paper the notions of solvable right transversal and nilpotent right transversal are defined. Further, it is proved that if a corefree subgroup has a generating solvable transversal, then the whole group is solvable.
\end{abstract}

\section{Introduction}

Let $\mathrm{G}$ be a group and $\mathrm{H}$ a proper subgroup of $\mathrm{G}$. A normalized right transversal is a subset of $\mathrm{G}$ obtained by selecting one and only one element from each right coset of $\mathrm{H}$ in $\mathrm{G}$, including the identity from the coset $\mathrm{H}$. Now we will call it a transversal in place of normalized right transversal. Suppose that $S$ is a transversal of $\mathrm{H}$ in $\mathrm{G}$. We define an operation $\circ$ on $S$ as follows: for $x, y \in S$, $\{x \circ y\}:=S \cap H x y$. It is easy to check that $(S, \circ)$ is a right loop, that is the equation of the type $X \circ a=b, X$ is unknown and $a, b \in S$ has a unique solution in $S$, and $(S, \circ)$ has a two-sided identity. In [5], it has been shown that for each right loop there exists a pair $(G, H)$ such that $H$ is a core-free subgroup of the group $\mathrm{G}$ and the given right loop can be identified with a transversal of $H$ in $G$. Not all transversals of a subgroup generate the group. But for finite groups, it is proved by Cameron in [2], that if a subgroup is core-free, then always there exists a transversal which generates the whole group. We call such a transversal a generating transversal.

2010 Mathematics Subject Classification: 20D99, 20N05

Key words and phrases: right loop, right transversal, solvable right loop, nilpotent right loop 
Let $(S, \circ)$ be a right loop (identity denoted by 1$)$. Let $x, y, z \in S$. Define a map $R(y, z)$ from $S$ to $S$ as follows: $R(y, z)(x)$ is the unique solution of the equation $X \circ(y \circ z)=(x \circ y) \circ z$, where $X$ is unknown. It is easy to verify that $R(y, z)$ is a bijective map. For a set $X$, let $\operatorname{Sym}(X)$ denote the symmetric group on $X$. We denote by $R \operatorname{Inn}(S)$ the subgroup of $\operatorname{Sym}(S)$, generated by the set $\{R(y, z) \mid y, z \in S\}$. This group is called the right inner mapping group of the right loop $S$. It measures the deviation of a right loop from being a group. Also note that right multiplication $R_{s}$ by an element $s \in S$ gives a bijective map from $S$ to $S$. The subgroup generated by $\left\{R_{S} \mid s \in S\right\}$ is the right multiplication group $\mathrm{RMlt}(\mathrm{S})$ of the right loop $\mathrm{S}$. One should note that the right multiplication group $R M l t(S)$ factorizes as $R \operatorname{Inn}(S) R(S)$ in $\operatorname{Sym}(S)$, where $R(S)=\left\{R_{s} \mid s \in S\right\}$. We will follow the right action convention for the map, that is the image of an element $x$ under a map $f$ is denoted by $x f$. Note that if $\mathrm{H}$ is a core-free subgroup of a group $\mathrm{G}$ and $\mathrm{S}$ is a generating transversal of $H$ in $G$, then $G \cong \operatorname{RMlt}(S)$ such that $H \cong \operatorname{RInn}(S)$ (see [6, Lemma, p. 1343]).

A non-empty subset $\mathrm{T}$ of right loop $\mathrm{S}$ is called a right subloop of $\mathrm{S}$, if it is right loop with respect to induced binary operation on $\mathrm{T}$ ([7, Definition $2.1, \mathrm{p}$. 2683]). An equivalence relation $R$ on a right loop $S$ is called a congruence in $S$, if it is a right subloop of $\mathrm{S} \times \mathrm{S}$. Also an invariant right subloop of a right loop $S$ is precisely the equivalence class of the identity of a congruence in $S$ ([7, Definition 2.8, p. 2689]). It is observed in the proof of [7, Proposition 2.10, p. 2690] that if $T$ is an invariant right subloop of $S$, then the set $S / T=\{T \circ x \mid x \in S\}$ becomes right loop called as quotient of $S \bmod T$. Let $\mathrm{R}$ be the congruence associated to an invariant right subloop $T$ of $S$. Then we also denote $S / T$ by $\mathrm{S} / \mathrm{R}$.

\section{Some properties of right loops}

In this section, we will recall some basic facts about right loops and also prove some of the results which will be used in next sections. Let $(S, \circ, /, 1)$ be a right loop, with right division / and two-sided identity 1 . Then $(S, \circ, /, 1)$ is Mal'tsev algebra with Mal'tsev term $\mathrm{P}(x, y, z)=(x / y) \circ z$.

The proof of the following Fundamental Theorem of homomorphism for right loops is as usual.

Proposition 1 Let $\rho: S \rightarrow S^{\prime}$ be a homomorphism of right loops. Then there exists a unique injective homomorphism $\bar{\rho}: S / \operatorname{Ker} \rho \rightarrow S^{\prime}$ such that $\bar{\rho} \circ v=\rho$, where $v: \mathrm{S} \rightarrow \mathrm{S} / \mathrm{Ker} \rho$ is the natural homomorphism. 
Lemma 1 Let $\mathrm{G}$ be a group, $\mathrm{H}$ a subgroup of $\mathrm{G}$ and $\mathrm{S}$ a transversal of $\mathrm{H}$ in $\mathrm{G}$. Suppose that $\mathrm{N} \unlhd \mathrm{G}$ containing $\mathrm{H}$. Then

$$
\mathrm{G} / \mathrm{N}=\mathrm{HS} / \mathrm{N} \cong \mathrm{S} / \mathrm{N} \cap \mathrm{S} .
$$

Proof. Suppose that $\circ$ denotes the induced right loop operation on $S$. Consider the map $\psi: S \rightarrow H S / N$ defined as $x \psi=x N$. This is a homomorphism, for

$$
\begin{aligned}
(x \circ y) \psi & =(x \circ y) N \\
& =h x y N \text { for some } h \in H \\
& =(x) \psi(y) \psi(H \subseteq N) .
\end{aligned}
$$

Also, $\operatorname{Ker} \psi=\{x \in S \mid x N=N\}=S \cap N$. Since for $h \in H$ and $x \in S$, we have $h x N=x N$ and $x \psi=x N, \psi$ is onto and so by Proposition $1, S / N \cap S \cong$ $\mathrm{HS} / \mathrm{N}$.

Let $\mathrm{G}$ be a group, $\mathrm{H}$ a subgroup and $\mathrm{S}$ a transversal of $\mathrm{H}$ in $\mathrm{G}$. Suppose that $\circ$ is the induced right loop structure on $\mathrm{S}$. We define a map $f: \mathrm{S} \times \mathrm{S} \rightarrow \mathrm{H}$ as: for $x, y \in S, f(x, y):=x y(x \circ y)^{-1}$. We further define the action $\theta$ of $H$ on $S$ as $\{x \theta h\}:=S \cap H x h$ where $h \in H$ and $x \in S$. Identifying $S$ with the set $H \backslash G$ of all right cosets of $\mathrm{H}$ in $\mathrm{G}$, we get a transitive permutation representation $\chi_{S}: G \rightarrow \operatorname{Sym}(S)$ defined by $\left\{(x)(g) \chi_{S}\right\}=S \cap H x g, g \in G, x \in S$. The kernel ker $\chi_{S}$ of this action is Core $_{G}(\mathrm{H})$, the core of $\mathrm{H}$ in $\mathrm{G}$.

One can check that $(\langle S\rangle \cap H) \chi_{S} \cong \operatorname{RInn}(S)$, where $\langle S\rangle$ denotes the subgroup of $G$ generated by $S$. Since $\chi_{S}$ is injective on $S$ and if we identify $S$ with $(S) \chi_{S}$, then $(\langle S\rangle) \chi_{S} \cong \operatorname{RMlt}(S)$. One can also verify that $\operatorname{ker}\left(\left.\chi_{S}\right|_{\langle S\rangle}:\langle S\rangle \rightarrow \operatorname{RMlt}(S)\right)=$ $\operatorname{ker}\left(\left.\chi_{S}\right|_{\langle S\rangle \cap H}:\langle S\rangle \cap H \rightarrow R \operatorname{Rnn}(S)\right)=$ Core $_{\langle S\rangle}(\langle S\rangle \cap H)$ and $\left.\chi_{S}\right|_{S}=$ the identity map on $S$.

With these notations it is easy to prove following lemma.

Lemma 2 For $x, y, z \in S$, we have $x \mathrm{R}(\mathrm{y}, z)=x \theta f(y, z)$.

Lemma 3 Let $\mathrm{H}$ be a subgroup of a group $\mathrm{G}$ and $\mathrm{S}$ a transversal of $\mathrm{H}$ in $\mathrm{G}$. Let $\mathrm{U}$ be a congruence on $\mathrm{S}$ considered as a right loop such that $\{(\mathrm{x}, \mathrm{x} \theta \mathrm{h}) \mid \mathrm{h} \in$ $\mathrm{H}, \mathrm{x} \in \mathrm{S}\} \subseteq \mathrm{U}$. Let $\mathrm{T}$ be the equivalence class of 1 under $\mathrm{U}$. Then $\mathrm{S} / \mathrm{U}$ is a group. Moreover, $\mathrm{N}=\mathrm{HT} \unlhd \mathrm{HS}=\mathrm{G}$ (and so $\mathrm{H} \leq \mathrm{N}$ and $\mathrm{N} \cap \mathrm{S}=\mathrm{T}$ ) and $\mathrm{G} / \mathrm{N} \cong \mathrm{S} / \mathrm{U}$.

Proof. Let $R$ be a congruence on $S$ generated by $\{(x, x R(y, z)) \mid x, y, z \in S\}$. Then, clearly $R \subseteq U$ and $S / U$ is a group. Let $\phi: G \rightarrow S / U$ be the map defined 
by $(h x) \phi=T \circ x, h \in H, x \in S$. This is a homomorphism, because for all $h_{1}, h_{2} \in H$ and $x_{1}, x_{2} \in S$,

$$
\begin{aligned}
\left(h_{1} x_{1} h_{2} x_{2}\right) \phi & =\left(h_{1} h\left(x_{1} \theta h_{2} \circ x_{2}\right)\right) \phi \text { for some } h \in H \\
& =T \circ\left(x_{1} \theta h_{2} \circ x_{2}\right) \\
& =\left(T \circ x_{1}\right) \circ\left(T \circ x_{2}\right) \quad\left(\text { for }\left(x_{1}, x_{1} \theta h_{2}\right) \in U\right) \\
& =\left(h_{1} x_{1}\right) \phi\left(h_{2} x_{2}\right) \phi .
\end{aligned}
$$

Let $h \in H$ and $x \in S$. Then $h x \in \operatorname{Ker} \phi$ if and only if $x \in \mathrm{T}$. Hence $\operatorname{Ker} \phi=$ $\mathrm{HT}=\mathrm{N}$ (say). This proves the lemma.

\section{Solvable right loops}

In this section, we will define a solvable right loop and obtain some of its properties.

Definition 1 A right loop $\mathrm{S}$ is said to be a solvable right loop if it has a finite composition series with abelian group factors.

Definition 2 Let $\mathrm{S}$ be a transversal of a subgroup $\mathrm{H}$ of $\mathrm{G}$. We call $\mathrm{S}$ a solvable transversal if it is solvable with respect to the induced right loop structure.

We define $S^{(1)}$ to be the smallest invariant right subloop of $S$ such that $S / S^{(1)}$ is an abelian group. We define $S^{(n)}$ by induction. Suppose $S^{(n-1)}$ is defined. Then $S^{(n)}$ is an invariant right subloop of $S$ such that $S^{(n)}=\left(S^{(n-1)}\right)^{(1)}$.

Theorem 1 If a group has a solvable generating transversal with respect to a core-free subgroup, then the group is solvable.

Proof. Let $\mathrm{G}$ be a group and $\mathrm{H}$ a core-free subgroup of it. Suppose that $\mathrm{S}$ is a generating transversal of $\mathrm{H}$ in $\mathrm{G}$. Then the group $\mathrm{G}$ can be written as $\mathrm{HS}$. By Lemma $1, \mathrm{G} / \mathrm{HG}^{(1)} \cong \mathrm{S} / \mathrm{S} \cap \mathrm{HG}^{(1)}$. So,

$$
\mathrm{S}^{(1)} \subseteq \mathrm{S} \cap \mathrm{HG}^{(1)} .
$$

By Lemma 3, $\mathrm{HS}^{(1)}$ is a normal subgroup of $\mathrm{G}$. Thus $\mathrm{G} / \mathrm{HS}^{(1)}=\mathrm{S} / \mathrm{S}^{(1)}$ (Lemma 1). Since $S / S^{(1)}$ is abelian, $\mathrm{G}^{(1)} \subseteq \mathrm{HS}^{(1)}$. Thus

$$
\mathrm{S} \cap \mathrm{HG}^{(1)} \subseteq \mathrm{S}^{(1)} .
$$


From (2) and (1), it is clear that

$$
\mathrm{S} \cap \mathrm{HG}^{(1)}=\mathrm{S}^{(1)}
$$

and

$$
\mathrm{HG}^{(1)}=\mathrm{HS}^{(1)} \text {. }
$$

We will use induction to prove that $H S^{(\mathfrak{n})}=H\left(H S^{(n-1)}\right)^{(1)}$ for $n \geq 1$. Define $\mathrm{S}^{(0)}=\mathrm{S}$. For $\mathrm{n}=1, \mathrm{HS}^{(1)}=\mathrm{HG}^{(1)}=\mathrm{H}\left(\mathrm{HS}^{(0)}\right)^{(1)}$ (by (4)). By induction, suppose that $H S^{(n-1)}=H\left(H S^{(n-2)}\right)^{(1)}$.

Since $S^{(n-1)} / S^{(n)} \cong H S^{(n-1)} / H S^{(n)}$ is an abelian group, $\left(H S^{(n-1)}\right)^{(1)} \subseteq H S^{(n)}$. Thus $\mathrm{H}\left(\mathrm{HS}^{(\mathrm{n}-1)}\right)^{(1)} \subseteq \mathrm{HS}^{(\mathrm{n})}$.

Further, $\mathrm{HS}^{(\mathrm{n}-1)} / \mathrm{H}\left(\mathrm{HS}^{(\mathrm{n}-1)}\right)^{(1)} \cong \mathrm{S}^{(\mathrm{n}-1)} /\left(\mathrm{S}^{(\mathrm{n}-1)} \cap \mathrm{H}\left(\mathrm{HS}^{(\mathrm{n}-1)}\right)^{(1)}\right)$ by Lemma 1. So $S^{(\mathfrak{n})} \subseteq S^{(n-1)} \cap H\left(H S^{(n-1)}\right)^{(1)}=S \cap H\left(H S^{(n-1)}\right)^{(1)}$. That is,

$$
H S^{(n)}=H\left(H S^{(n-1)}\right)^{(1)} \text { for all } n \geq 1 .
$$

Now (5) implies that

$$
H S^{(n)}=H\left(H S^{(n-1)}\right)^{(1)} \supseteq H\left(H\left(H S^{(n-2)}\right)^{(1)}\right)^{(1)} \supseteq H\left(H S^{(n-2)}\right)^{(2)} .
$$

Proceeding inductively, we have $H S^{(\mathfrak{n})} \supseteq \mathrm{H}(H S)^{(\mathfrak{n})}=\mathrm{HG}^{(\mathrm{n})}$. Suppose that $\mathrm{S}$ is a solvable right loop, that is there exists $n \in \mathbb{N}$ such that $S^{(\mathfrak{n})}=\{1\}$. Then $\mathrm{G}^{(\mathfrak{n})} \subseteq \mathrm{H}$. Since $\mathrm{G}^{(\mathfrak{n})}$ is a normal subgroup of $\mathrm{G}$ contained in $\mathrm{H}$, so $\mathrm{G}^{(\mathfrak{n})}=\{1\}$. This proves the theorem.

The converse of the above theorem is not true. For example take $G$ to be the symmetric group on three symbols and $\mathrm{H}$ to be any two order subgroup of it. Then $\mathrm{H}$ has no solvable generating transversal but we know that $\mathrm{G}$ is solvable. Following is an easy consequence of the above theorem.

Corollary 1 The right multiplication group of a solvable right loop is a solvable group.

\section{Nilpotent right loops}

In this section, we define nilpotent right loops as a special case of the nilpotent Mal'tsev algebras defined in [8]. We will obtain some properties of nilpotent right loops. This will generalize a result of [1].

Definition 3 [8, Definition 211, p. 24] Let $\beta$ and $\gamma$ be congruences on a right loop $\mathrm{S}$. Let $(\gamma \mid \beta)$ be a congruence on $\beta$. Then $\gamma$ is said to centralize $\beta$ by means of the centering congruence $(\gamma \mid \beta)$ such that following conditions are satisfied: 
(i) $(x, y)(\gamma \mid \beta)(u, v) \Rightarrow x \gamma u$, for all $(x, y),(u, v) \in \beta$.

(ii) For all $(x, y) \in \beta$, the map $\pi:(\gamma \mid \beta)_{(x, y)} \rightarrow \gamma_{x}$ defined by $(\mathfrak{u}, v) \mapsto u$ is a bijection, where for a set $\mathrm{X}$ and an equivalence relation $\delta$ on $\mathrm{X}, \delta_{w}$ denotes the equivalence class of $w \in X$ under $\delta$.

(iii) For all $(x, y) \in \gamma,(x, x)(\gamma \mid \beta)(y, y)$.

(iv) $(x, y)(\gamma \mid \beta)(u, v) \Rightarrow(y, x)(\gamma \mid \beta)(v, u)$, for all $(x, y),(u, v) \in \beta$.

(v) $(x, y)(\gamma \mid \beta)(u, v)$ and $(y, z)(\gamma \mid \beta)(v, w) \Rightarrow(x, z)(\gamma \mid \beta)(u, w)$, for all $(x, y),(u, v),(y, z)$ and $(v, w)$ in $\beta$.

By (i) and (iv), we observe that $(x, y)(\gamma \mid \beta)(u, v) \Rightarrow y \gamma \nu$.

Let $S$ be a right loop. If a congruence $\alpha$ on $S$ is centralized by $S \times S$, then it is called a central congruence (see [8, p. 42]). By [8, Proposition 221, p. 34] and [8, Proposition 226, p. 38], there exists a unique maximal central congruence $\zeta(S)$ on $S$, called as the center congruence of $S$. For a right loop, it is product of all centralizing congruences. The center $\mathcal{Z}(S)$ of $S$ is defined as $\zeta_{1}$, the equivalence class of the identity 1 . In [4, Proposition 3.3, p. 6], it is observed that if $x \in \mathcal{Z}(S)$, then $x \circ(y \circ z)=(x \circ y) \circ z$ for all $y, z \in S$. In [4, Proposition 3.4 , p. 6], it is observed that if $x \in \mathcal{Z}(S)$, then $x \circ y=y \circ x$ for all $y \in S$. This means that the center $\mathcal{Z}(S)$ is an abelian group.

Definition 4 A right loop $\mathrm{S}$ is said to be nilpotent if it has a central series

$$
\{1\}=\mathcal{Z}_{0} \leq \mathcal{Z}_{1} \leq \cdots \leq \mathcal{Z}_{\mathrm{n}}=\mathrm{S}
$$

for some $\mathrm{n} \in \mathbb{N}$, where

$$
\mathcal{Z}_{\mathrm{i}+1} / \mathcal{Z}_{\mathrm{i}}=\mathcal{Z}\left(\mathrm{S} / \mathcal{Z}_{\mathrm{i}}\right) \text { and } \mathcal{Z}_{1}=\mathcal{Z}(\mathrm{S}) .
$$

On can observe that $\mathcal{Z}_{i}(0 \leq i \leq n)$ is an invariant right subloop of $\mathrm{S}$. We call a transversal $S$ of a subgroup $\mathrm{H}$ of a group $\mathrm{G}$ to be nilpotent, if it is nilpotent with respect to the induced right loop structure.

Lemma 4 Every nilpotent right loop is a solvable right loop.

Proof. It follows from the fact that the central series of a nilpotent right loop is a composition series with abelian group factors.

Since a nilpotent right loop $S$ is solvable, by Corollary $1, \operatorname{RMlt}(S)$ is solvable. But in this proof we do not know much about the structure of $\operatorname{RInn}(S)$. We will obtain that if $S$ is a nilpotent right loop of prime power order, then the order of $\operatorname{RMlt}(S)$ will be a prime power. 
Proposition 2 Let $\mathrm{S}$ be a right loop. Let $\theta: \operatorname{RInn}(\mathrm{S}) \rightarrow \operatorname{RInn}(\mathrm{S} / \mathcal{Z}(\mathrm{S}))$ be the onto homomorphism induced by the natural projection $\vee: S \rightarrow S / \mathcal{Z}(S)$.

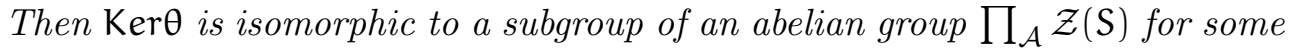
indexing set $\mathcal{A}$.

Proof. Let $\mathcal{A}=\left\{x_{1}, \cdots, x_{i}, \cdots\right\}$ be a set obtained by choosing one element from each right coset of $\mathcal{Z}(S)$ in $S$, with $x_{1}=1 \in \mathcal{Z}(S)$. Then $S=\sqcup_{x_{i} \in \mathcal{A}}(\mathcal{Z}(S) \circ$ $\left.x_{i}\right)$. Let $h \in \operatorname{Ker} \theta$. Then $x_{i} h=z \circ x_{i}$ for some $z \in \mathcal{Z}(S)$. If $y=u_{i} \circ x_{i}$, where $u_{i} \in \mathcal{Z}(S)$, then

$(y) h=\left(u_{i} \circ x_{i}\right) h=u_{i} \circ\left(x_{i}\right) h$ (by condition (C7) of [5, Definition 2.1, p. 71] and $[4$, Proposition 3.3 , p. 6])

$$
\begin{aligned}
& =u_{i} \circ\left(z \circ x_{i}\right) \\
& =\left(u_{i} \circ z\right) \circ x_{i}\left(\text { for } u_{i} \in \mathcal{Z}(S)\right) \\
& =\left(z \circ u_{i}\right) \circ x_{i} \\
& =z \circ\left(u_{i} \circ x_{i}\right)(\text { for } z \in \mathcal{Z}(S)) \\
& =z \circ y .
\end{aligned}
$$

Thus $\mathrm{h} \in \operatorname{Ker} \theta$ is completely determined by $x_{i} h\left(x_{i} \in \mathcal{A}\right)$. Therefore, it defines a map $\eta: \operatorname{Ker} \theta \rightarrow \prod_{\mathcal{A}} \mathcal{Z}(S)$ by $(h) \eta=\left(z_{i}\right)_{\mathcal{A}}$, where $\left(x_{i}\right) h=z_{i} \circ x_{i}$. One can check that $\eta$ is injective homomorphism.

Following is the finite version of above proposition.

Corollary 2 Let $\mathrm{S}$ be a finite right loop with $|\mathrm{S} / \mathcal{Z}(\mathrm{S})|=\mathrm{k}$. Let $\theta: \mathrm{RInn}(\mathrm{S}) \rightarrow$ $\operatorname{RInn}(\mathrm{S} / \mathcal{Z}(\mathrm{S})$ ) be the onto homomorphism induced by natural projection $v$ : $\mathrm{S} \rightarrow \mathrm{S} / \mathcal{Z}(\mathrm{S})$. Then $\operatorname{Ker} \theta$ is isomorphic to a subgroup of abelian group $\mathcal{Z}(\mathrm{S}) \times$ $\cdots \times \mathcal{Z}(\mathrm{S})(\mathrm{k}-1$ times $)$.

Let $S$ be a nilpotent right loop with central series

$$
\{1\}=\mathcal{Z}_{0} \leq \mathcal{Z}_{1} \leq \cdots \leq \mathcal{Z}_{\mathrm{n}}=\mathrm{S} .
$$

Let $\theta_{j}: \operatorname{RInn}(S) \rightarrow \operatorname{RInn}\left(S / \mathcal{Z}_{j}\right)(0 \leq j \leq n-1)$ be onto homomorphism induced by the natural projection $v_{j}: S \rightarrow S / \mathcal{Z}_{j}$. Then this will give a series

$$
\{1\}=\operatorname{Ker} \theta_{0} \leq \cdots \leq \operatorname{Ker} \theta_{n-1}=\operatorname{RInn}(S) .
$$

Let $\theta: \operatorname{RInn}\left(S / \mathcal{Z}_{j}\right) \rightarrow \operatorname{RInn}\left(\left(S / \mathcal{Z}_{j}\right) /\left(\mathcal{Z}_{j+1} / \mathcal{Z}_{j}\right)\right)$ be onto homomorphism induced by the natural projection $v: S / \mathcal{Z}_{j} \rightarrow\left(S / \mathcal{Z}_{j}\right) /\left(\mathcal{Z}_{j+1} / \mathcal{Z}_{j}\right)$. By Proposition 2 , $\operatorname{Ker} \theta$ is isomorphic to a subgroup of $\prod_{\mathcal{B}} \mathcal{Z}_{j+1} / \mathcal{Z}_{j}$ for some indexing set $\mathcal{B}$.

We now observe that each member of $\operatorname{Ker} \theta_{j+1} / \operatorname{Ker} \theta_{j}$ induces a member of $\operatorname{Ker} \theta$. For this, we will see the action of an element of $\operatorname{Ker} \theta_{j+1} / \operatorname{Ker} \theta_{j}$ on the 
elements of $\left(\mathrm{S} / \mathcal{Z}_{\mathfrak{j}}\right) /\left(\mathcal{Z}_{\mathfrak{j}+1} / \mathcal{Z}_{\mathfrak{j}}\right)$. Let $h_{j+1} \operatorname{Ker} \theta_{j} \in \operatorname{Ker} \theta_{j+1} / \operatorname{Ker} \theta_{j}$, where $h_{j+1} \in$ $\operatorname{Ker} \theta_{j+1}$ and $\left(\mathcal{Z}_{j+1} / \mathcal{Z}_{j}\right) \circ\left(\mathcal{Z}_{j} \circ x\right) \in\left(S / \mathcal{Z}_{j}\right) /\left(\mathcal{Z}_{j+1} / \mathcal{Z}_{j}\right)$. By the definition of $\theta_{j}$, each element of $\operatorname{Ker} \theta_{j}$ acts trivially on the cosets of $\mathcal{Z}_{j}$. Since $\operatorname{RInn}\left(S / \mathcal{Z}_{j+1}\right)$ $\cong \operatorname{RInn}\left(\left(S / \mathcal{Z}_{j}\right) /\left(\mathcal{Z}_{j+1} / \mathcal{Z}_{j}\right)\right)$, by definition of $\theta_{j+1}, h_{j+1}$ also acts trivially on $\left(\mathcal{Z}_{j+1} / \mathcal{Z}_{j}\right) \circ\left(\mathcal{Z}_{j} \circ x\right)$. Thus, we have proved the following:

Proposition 3 Let $\mathrm{S}$ be a nilpotent right loop with central series \%. Then there exists a series

$$
\{1\}=\operatorname{Ker} \theta_{0} \leq \cdots \leq \operatorname{Ker} \theta_{n-1}=\operatorname{RInn}(S)
$$

such that $\operatorname{Ker} \theta_{j+1} / \operatorname{Ker} \theta_{j}$ is isomorphic to a subgroup of $\prod_{\mathcal{B}} \mathcal{Z}_{j+1} / \mathcal{Z}_{j}$ for some indexing set $\mathcal{B}$.

Corollary 3 Let $\mathrm{S}$ be a nilpotent right loop. Then the right inner mapping group $\mathrm{RInn}(\mathrm{S})$ is a solvable group.

Proof. By Proposition 3, central series of $S$ gives a series of $\operatorname{RInn}(S)$ with abelian quotients.

Corollary 4 If a group $\mathrm{G}$ has a nilpotent generating transversal with respect to a core-free subgroup $\mathrm{H}$, then $\mathrm{H}$ is solvable.

Corollary 5 Let $\mathrm{S}$ be a nilpotent generating transversal with respect to a corefree subgroup $\mathrm{H}$ of a finite group $\mathrm{G}$ such that $|\mathrm{S}|=\mathrm{p}^{\mathrm{n}}$ for some prime $\mathrm{p}$ and $\mathrm{n} \in \mathbb{N}$. Then both $\mathrm{H}$ and $\mathrm{G}$ are $\mathrm{p}$-groups.

\section{Some examples}

In this section, we will observe some examples and counterexamples. We have seen that the concepts of solvability and nilpotency of a right loop can be transferred in term of a generating transversal of a core-free subgroup of a group. There are examples of groups where no non-trivial subgroup is corefree. Following is an example of such a group:

Example 1 Consider the group $\mathrm{G}=\left\langle\mathrm{x}_{1}, \mathrm{x}_{2}, \mathrm{x}_{3}, \mathrm{x}_{4}\right| \mathrm{x}_{1}^{\mathrm{p}^{\mathrm{n}}}=x_{2}^{\mathrm{p}^{3}}=x_{3}^{\mathrm{p}^{2}}=x_{4}^{\mathrm{p}^{2}}=1$, $\left[x_{1}, x_{2}\right]=x_{2}^{p^{2}},\left[x_{1}, x_{3}\right]=x_{3}^{p},\left[x_{1}, x_{4}\right]=x_{4}^{p},\left[x_{2}, x_{3}\right]=x_{1}^{p^{n-1}},\left[x_{2}, x_{4}\right]=x_{2}^{p^{2}},\left[x_{3}, x_{4}\right]$ $\left.=x_{4}^{p}\right\rangle$ where $\mathrm{p}$ is an odd prime and $\mathrm{n}$ is the natural number greater than 2 . The above example has been taken from [3]. This is a nilpotent group of class 2 having no nontrivial core-free subgroup. 
We now observe that a solvable right loop which is not a group need not be a nilpotent right loop.

Example 2 Let $\mathrm{G}=\operatorname{Alt}(4)$, the alternating group of degree 4 and $\mathrm{H}=$ $\{\mathrm{I},(1,2)(3,4)\}$, where I denotes the identity permutation. Consider a right transversal $\mathrm{S}=\{\mathrm{I},(1,3)(2,4),(1,2,3),(1,3,2),(2,3,4),(1,3,4)\}$ of $\mathrm{H}$ in $\mathrm{G}$. Note that $\langle\mathrm{S}\rangle=\mathrm{G}$ and $\mathrm{H}$ is core-free. Then $\operatorname{RMlt}(\mathrm{S}) \cong \mathrm{G}$ and $\operatorname{RInn}(\mathrm{S}) \cong \mathrm{H}$. Also note that $\mathrm{S} \cap \mathrm{N}_{\mathrm{G}}(\mathrm{H})=\{\mathrm{I},(1,3)(2,4)\}$, where $\mathrm{N}_{\mathrm{G}}(\mathrm{H})$ denotes the normalizer of $\mathrm{H}$ in $\mathrm{G}$. By [4, Proposition 3.3, p. 6], $\mathcal{Z}(\mathrm{S}) \subseteq \mathrm{S} \cap \mathrm{N}_{\mathrm{G}}(\mathrm{H})$. Let $\circ$ be the induced binary operation on $\mathrm{S}$ as defined in the Section 1. Observe that $(1,3)(2,4) \circ(1,3,4) \neq(1,3,4) \circ(1,3)(2,4)$. This implies that $\mathcal{Z}(S)=\{\mathrm{I}\}$. Hence $\mathrm{S}$ can not be nilpotent.

Now by Lemma 1, $\mathrm{S} /\left(\mathrm{S} \cap \mathrm{N}_{\mathrm{G}}(\mathrm{H})\right)$ is isomorphic to the cyclic group of order 3. This implies that $\mathrm{S}$ is solvable.

Now, we observe that, unlike for the case of groups, a right loop of prime power order need not be nilpotent.

Example 3 Let $\mathrm{G}=$

$$
\langle(1,3)(2,4)(5,7,6,8),(1,4)(2,3)(5,8,6,7),(1,5)(2,6)(3,7)(4,8)\rangle \leq \operatorname{Sym}(8),
$$

where $\operatorname{Sym}(\mathrm{n})$ denotes the symmetric group of degree $\mathrm{n}$. Let $\mathrm{H}$ be the stabilizer of 1 in $\mathrm{G}$. Consider $S=\{\mathrm{I},(1,2)(3,4),(1,3)(2,4)(5,7,6,8),(1,4)(2,3)$ $(5,8,6,7), \quad(1,5)(2,6)(3,7)(4,8), \quad(1,6)(2,5)(3,8)(4,7), \quad(1,7)(2,8)(3,6,4,5)$, $(1,8)(2,7)(3,5,4,6)\}$. Clearly $\mathrm{S}$ is right transversal of $\mathrm{H}$ in $\mathrm{G}$. Note that the center $\mathrm{Z}(\mathrm{G})=\{\mathrm{I},(1,2)(3,4)(5,6)(7,8)\}$ and $\mathrm{N}_{\mathrm{G}}(\mathrm{H})=\mathrm{HZ}(\mathrm{G})$. Since $\mathrm{H}$ is corefree and $\langle\mathrm{S}\rangle=\mathrm{G}, \mathrm{G} \cong \operatorname{RMlt}(\mathrm{S})$ and $\mathrm{H} \cong \operatorname{RInn}(\mathrm{S})$. Observe that $\mathrm{S} \cap \mathrm{N}_{\mathrm{G}}(\mathrm{H})=$ $\{\mathrm{I},(1,2)(3,4)\}$. By [4, Proposition 3.3, p. 6], $\mathcal{Z}(\mathrm{S}) \subseteq \mathrm{S} \cap \mathrm{N}_{\mathrm{G}}(\mathrm{H})$. Let $\circ$ be the induced binary operation on $S$ as defined in the section 1 . Observe that $(1,2)(3,4) \circ(1,5)(2,6)(3,7)(4,8) \neq(1,5)(2,6)(3,7)(4,8) \circ(1,2)(3,4)$. This implies that $\mathcal{Z}(\mathrm{S})=\{\mathrm{I}\}$. Hence $\mathrm{S}$ cannot be nilpotent.

Next, we will show that there are core-free subgroups of a nilpotent group which has none of its generating transversals nilpotent. But before proceeding to further examples, we need to prove the following results.

Proposition 4 Suppose that $\mathrm{G}$ is a nilpotent group of class 2, $\mathrm{H}$ is a core-free subgroup of $\mathrm{G}$ and $\mathrm{S}$ is generating transversal of $\mathrm{H}$ in $\mathrm{G}$. Then $\mathcal{Z}(\mathrm{G}) \cap \mathrm{S}=$ $\mathcal{Z}(\mathrm{S})$. 
Proof. Take $x \in \mathcal{Z}(S)$. Then $x \circ y=y \circ x$ for all $y \in S$. This implies $x y x^{-1} y^{-1} \in$ $\mathrm{H}$ for all $\mathrm{y} \in \mathrm{S}$. Since group is nilpotent of class 2, so all commutators are central. For $\mathrm{H}$ is core-free, so $\mathrm{H}$ will not contain any commutator element. This implies $x y x^{-1} y^{-1}=1$ or $x y=y x$ for all $y \in S$. This proves that $\mathcal{Z}(S) \subseteq$ $\mathcal{Z}(\mathrm{G}) \cap S$ (for $S$ generates $\mathrm{G}$ ). Converse is obvious. This proves the lemma.

Proposition 5 For some prime $\mathrm{p}$, suppose that $\mathrm{G}$ is a $\mathrm{p}$-group of nilpotent class $2, \mathrm{H}$ is a core-free subgroup of $\mathrm{G}$ and $\mathrm{S}$ is generating transversal of $\mathrm{H}$ in $\mathrm{G}$. Then $\mathcal{Z}(\mathrm{G}) \cap \Phi(\mathrm{G}) \cap \mathrm{S}=\{1\}$ where $\Phi(\mathrm{G})$ is the Frattini subgroup of $\mathrm{G}$.

Proof. Suppose that $1 \neq x \in \mathcal{Z}(\mathrm{G}) \cap \Phi(\mathrm{G}) \cap S$. Then by Proposition $4, x \in$ $\mathcal{Z}(\mathrm{S})$. Also $\mathcal{Z}(\mathrm{S})$ is an invariant right subloop, so $|\mathcal{Z}(\mathrm{S})|$ divides $|\mathrm{S}|$. Consider $S^{\prime}=S \backslash\{x\} \cup\{h x\}$ for some $1 \neq h \in H$. Note that $S^{\prime}$ also generates $G$. Then by Proposition 4 , order of center of $S^{\prime}$ is one less than the order of center of $S$ and also $\left|\mathcal{Z}\left(S^{\prime}\right)\right|$ divides $|S|$. This is not possible for order of $S$ is $p$ power. This proves the lemma.

Example 4 Consider the group $\mathrm{G}=\left\langle\mathrm{x}_{1}, \mathrm{x}_{2}, \mathrm{x}_{3}, \mathrm{x}_{4}\right| x_{1}^{\mathrm{p}^{\mathrm{n}}}=x_{2}^{\mathrm{p}^{2}}=x_{3}^{\mathrm{p}^{2}}=x_{4}^{\mathrm{p}^{4}}=$ $1,\left[x_{1}, x_{2}\right]=x_{2}^{p},\left[x_{1}, x_{3}\right]=x_{3}^{p},\left[x_{1}, x_{4}\right]=x_{3}^{p},\left[x_{2}, x_{3}\right]=x_{1}^{p^{n-1}},\left[x_{2}, x_{4}\right]=x_{2}^{p},\left[x_{3}, x_{4}\right]=$ 1) where $\mathrm{p}$ is an odd prime. The above example has been taken from [3]. By the Lemma 2.1 of [3], this group is a nilpotent group of of class 2 and its center and Frattini subgroup are equal. By Propositions 4 and 5, it follows that center of each generating transversal is trivial. So none of the generating transversal is nilpotent.

\section{Acknowledgments}

The authors are grateful to the referee for their valuable comments and suggestions for the improvement of the paper. Second author is supported by UGC-BSR Research Start-up-Grant No. F.-2(20)/2012(BSR).

\section{References}

[1] R. H. Bruck, Contributions to the Theory of Loops, Trans. Amer. Math. Soc., 60 (1946), 245-354.

[2] P. J. Cameron, Generating a group by a transversal, Preprint available at http://www.maths.qmul.ac.uk/ pjc/preprints/transgenic.pdf 
[3] V. K. Jain, M. K. Yadav, On finite p-groups whose automorphisms are all central, Israel J. Math., 189 (2012), 225-236.

[4] V. Kakkar, R. P. Shukla, On the Congruences in Right Loops, Comm. Algebra, 43 (12) (2015), 5121-5130.

[5] R. Lal, Transversals in Groups, J. Algebra, 181 (1996), 70-81.

[6] J. D. Phillips, Right quotients of groups, Comm. Algebra, 25 (4) (1997), 1341-1345.

[7] R. P. Shukla, Congruences in right quasigroups and general extensions, Comm. Algebra, 23 (1995), 2679-2695.

[8] J. D. H. Smith, Mal'cev Varieties, Springer Lecture Notes in Mathematics, Number 554, (1976).

[9] J. D. H. Smith, A. B. Romanowska, Post Modern Algebra, John Wiley \& Sons, Inc., (1999). 